\title{
Ileoanal J-Pouch prolapse: successful laparoscopic pouchopexy with a biological mesh
}

\begin{abstract}
Background and aims: Prolapse of ileal pouch is uncommon complication and the surgical treatment of this entity is very challenging. Only a few reports in the literature describe the repair of pouch prolapse with synthetic material, and there is no report describing the use of a biological mesh.

\section{Methods: Case report}

Results: A 34-year old female was diagnosed with colitis ulcerosa at the age of 29. One year after she had a subtotal colectomy and two years later, she underwent completion proctectomy with an ileo-anal J pouch (IPAA). Both procedures were performed by open approach. The patient developed a recurrent full-thickness prolapse of the pouch that failed the initial nonoperative management and she was successfully treated with laparoscopic pouchopexy, using a biological mesh.
\end{abstract}

Conclusion: Laparoscopic pouchopexy with a biological mesh appears to be a reasonable option in the surgical management of a full-thickness prolapse of ileoanal J-pouch prolapse.
Volume 7 Issue I - 2017

\author{
Kristin Julia Steinthorsdottir,' Orhan Bulut ${ }^{2}$ \\ 'Department of Gastrointestinal Surgery, Copenhagen \\ University Hospital Hvidovre, Denmark \\ ${ }^{2}$ Institute of Clinical Medicine, University of Copenhagen, \\ Denmark
}

Correspondence: Orhan Bulut, Department of Surgical Gastroenterology, Copenhagen University Hospital Hvidovre, Kettegaards allé 30, DK-2650 Hvidovre, Denmark, Tel +45 3862 695I, Fax +453862 3760, Email Orhan.Bulut@hvh.regionh.dk

Received: May 10,2017 | Published: June 27, 2017

\section{Introduction}

Proctocolectomy with ileal pouch-anal anastomosis (IPAA) has become the standard surgical treatment for patients with intractable ulcerative colitis, undergoing elective resections. This procedure can be performed by an open or laparoscopic approach and is associated with a good quality of life and long-term outcomes. ${ }^{1}$ However, there are several well-known complications of the procedure and the pouch itself. Common late complications include anastomotic strictures, residual proctitis/pouchitis and poor pouch function. ${ }^{2}$ Prolapse of the pouch on the other hand, is an uncommon complication, rarely described in the literature. Therefore the experience with management of the complication is very limited. The surgical treatments reported include transanal repair, transabdominal pouchopexy and transabdominal revision or removal. ${ }^{3,4}$ A single case-report describes a successful robotic-assisted laparoscopic pouchopexy. ${ }^{5}$ Only a few reports describe the repair of pouch prolapse with synthetic material, and there is no report describing the use of a biological mesh. Therefore we present our experience with a case of J-pouch prolapse repaired with a biologic mesh using a laparoscopic approach.

\section{Case report}

\section{Case history}

A 34-year old female was diagnosed with colitis ulcerosa at the age of 29. One year after she had a subtotal colectomy and two years later, she underwent completion proctectomy with an ileo-anal $\mathrm{J}$ pouch (IPAA). Both procedures were performed by open approach A few months after the operation, the patient reported recurrent fullthickness prolapse of the pouch when passing stool. In the beginning this was intermittent and the patient could reduce the prolapse manually or with pelvic floor exercises. At this point, the patient was not interested in operative management. Gradually however, the frequency and severity increased, with prolapse several times a day, and the patient had also required help for reduction, including several times under general anaesthesia. Endoscopy of the pouch was performed several times during the course. At one point there had been minimal pouchitis, but otherwise the endoscopies were normal.

Anorectal examinations revealed a normal sphincter apparatus, and pelvic floor evaluation with anorectal physiology examination determined normal function and medium strength. To optimize the protective effect of the pelvic floor muscles, the patient was instructed in pelvic muscles rehabilitation. This had minimal effect, and the recurrent prolapses had severe influence on the patient's day-to-day functions. It was decided to perform a laparoscopic pouchopexy, with the risk of converting to open surgery. The patient was informed about the procedure and possible complications and the informed consent was obtained.

\section{Operative management}

The patient was placed in Lloyd-Davis position. The abdominal cavity was entered by open method, by performing a cut-down procedure for camera port insertion. A total of four port sites were used, a $12 \mathrm{~mm}$ supraumbilical cameraport and three $5 \mathrm{~mm}$ ports. Laparoscopic exploration showed severe intraabdominal adhesions, especially concentrating in the pelvis between the pouch, the right ureter and the right uterine adnex.

The dissection was started by adhesiolysis between small intestinal loops and between the small intestine, the parietal abdominal/pelvic wall and internal genital organs. Multiple intra-loop small intestinal adhesions were lysed using mainly a monopolar laparoscopic scissor. Meticulous attention was given not to injure the intestinal wall. The small intestine was followed in a retrograde manner from the ileal pouch anastomosis with atraumatic bowel graspers. When gaining access to the pelvis, the dissection of dense adherences between the pouch and uterus, bilateral fallopian tubes and ovaries were completed to visualise major pelvic structures and the prolapsing segment of the pouch. There was a significant redundancy of the J-pouch with a very low peritoneal cul-de-sac. Also two uterine fibromas were noted. 
Using a bowel grasper, the upper part of the pouch was gently retracted cranially out of the pelvis, to allow for visualization of the sacral promontory and the vascular anatomy of this area. Both ureters were clearly identified. Then, the pre-sacral plane was entered and through careful dissection, the mesentery of the pouch was completely mobilised down to the level of the pelvic floor and the anastomosis. This plane is avascular, allowing for bloodless dissection down to levator ani. Hereafter the left-sided adherences between pouch and pelvic wall were incised and the pouch was retracted by the assistant to the patient's right side, to allow complete mobilisation of the pouch. The dissection was determined complete by digital exploration of the anal canal. Once the pouch mesentery was completely mobilised and retracted into anatomical position, a porcine dermal collagen mesh (Permacol ${ }^{\text {TM}}$, Tissue Science Laboratories, Aldershot, Hampshire, UK) was introduced through the camera port. The mesh was placed on the anterior surface of the sacrum, and secured to the promontory (Figure 1) and the sacrum with titanium tacks (Protack ${ }^{\mathbf{T M}}$, Covidien, Dublin, Ireland). The mesh covered 2/3 of the rectal circumference (Figure 2). After ensuring the reposition of the pouch, the side edges of the mesh were sutured to the lateral sides of the pouch with nonabsorbable prolene thread (Figure 3). The fibromas were also excided after advise from the gynaecological consultant during the same procedure.

$\mathrm{M}=$ Mesh $\mathrm{P}=$ pouch $\mathrm{U}=$ uterus $>=$ sutures

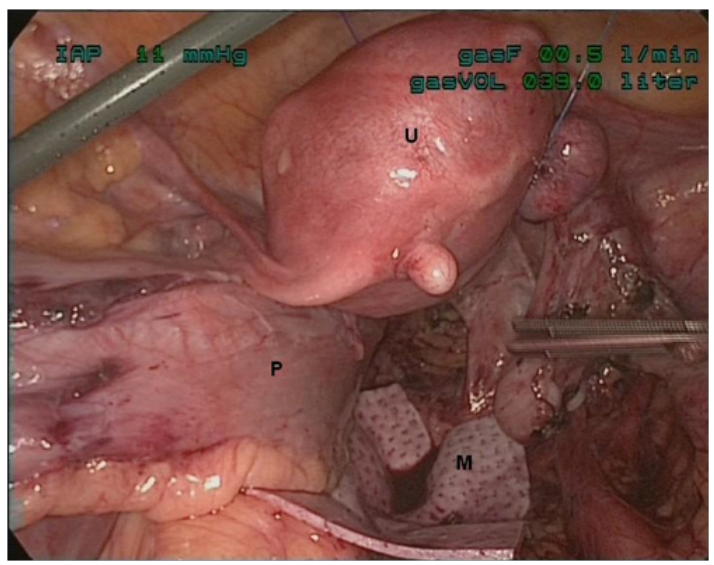

Figure I The biological mesh was placed through the presacral fasciae and ileal J-pouch.

$\mathrm{M}=$ Mesh $\mathrm{P}=$ pouch $\mathrm{U}=$ uterus

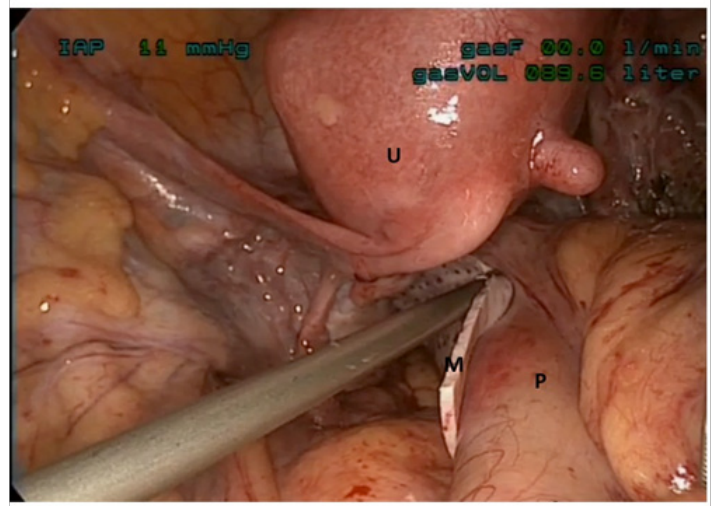

Figure 2 The view of the mesh position as seen from the left side at the sacral promontory.

$\mathrm{M}=$ Mesh $\mathrm{P}=$ pouch $\mathrm{U}=$ uterus

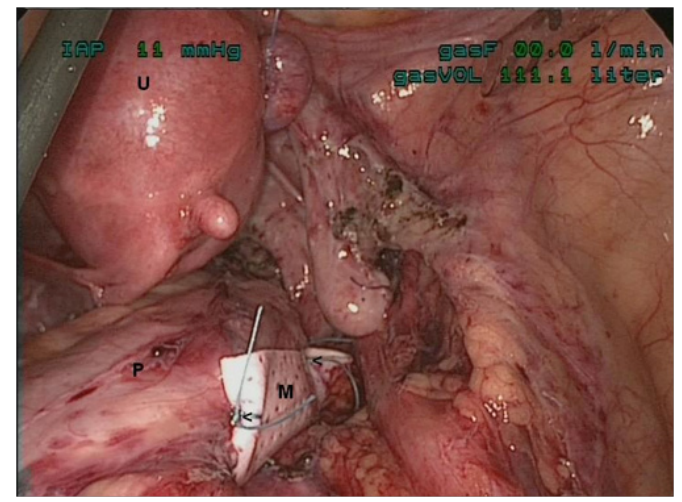

Figure 3 The final view of operative field after fixation of the biological mesh. The nonabsorbable sutures were used to attach the J-pouch to the mesh.

\section{Results}

The operation and the post-operative recovery were uneventful. The operative time was 257 minutes and the estimated blood loss was minimal. The patient followed a fast track recovery program, including epidural analgesia, full oral feeding, early removal of urinary catheter and early mobilization. She was discharged on the fifth post-operative day, and seen at the outpatient clinic for postoperative evaluation at 1 , 3,6 and 12 months following surgery. She was well, without episodes of pouch prolapse or fecal incontinence.

\section{Discussion}

Ileo anal-pouch prolapse, which was first reported in $1994^{6}$ is an uncommon complication to IPAA. Most pouch prolapses occur within two years of the original procedure. The pathogenesis is unknown, but there has been a theoretical consideration about the tension of pouch mesenterium. Patients presents with a sensation of incomplete evacuation, seepage, anal pain and incontinence. The largest material on the topic is a survey sent to all members of the American Society of Colon and Rectal Surgeons in 2001. Of 23,541 procedures reported, a total of 85 cases $(0.4 \%)$ presented with mucosal or full-thickness pouch prolapse (overall response rate was $19.5 \%$ (269 respondents)). Surgical treatment was performed in 52 patients through transanal, transabdominal or combined approach, with a success rate of 94 $\% .{ }^{4}$ A single-centre study reports similar numbers, with an overall incidence of $0.3 \%$ (11 out of 3,176 patients). Seven of these were full-thickness prolapse. Six patients were treated with transabdominal suture pouchopexy, and three of those had recurrence, and subsequent conversion of the pouch to an ileostomy. Due to the high recurrence rate, the seventh patient underwent transabdominal pouchopexy with a biological mesh. At nine months follow-up there was no recurrence. ${ }^{3}$

The latter study highlights the difficulties the surgeon meets when planning an operation for an ileo-anal pouch prolapse. Even when considering the more common rectal prolapse, no high evidence guidelines exist on the numerous surgical options. The current surgical options include transanal repair, abdominal pouchpexy and transabdominal revision. The laparoscopic approach has become the preferred procedure for the transabdominal management of fullthickness rectal prolapse. It is generally accepted, that the abdominal operations are preferred over perineal operations in fit patients, but there are no large randomized clinical trials to support the choice. ${ }^{6,7}$ Furthermore it is still debatable whether the rectopexy should be performed by sutures, synthetic or biological mesh. ${ }^{8}$ Based on the 
principle of rectopexy by adhesions and fibrosis, on the assumption that fixation using a mesh would be more effective than that using a simple suture, meshes and other prostheses have been developed. With minimal prior experience and very limited guidance from the literature, we chose to perform a laparoscopic pouchopexy with a biological mesh. The decision of which operative approach and method to use in this case was based on several considerations. We took into account, that the patient was young and fit with a long life expectancy, and that any recurrence would require another high-risk operation. In a retrospective study, comparing laparoscopic suture and mesh rectopexy, Sahoo et al., ${ }^{7}$ found no differences in regards to morbidity, recurrence or cost. ${ }^{7}$ Additionally, a systematic review of thirteen observational studies, found synthetic and biological mesh rectopexy comparable, with low recurrence and complication rates. ${ }^{8}$

As reported from Joyce et al., ${ }^{3}$ it is also our experience that the suture rectopexy has higher recurrence rates, and that the complicationand recurrence rates with biological meshes are low. The biological meshes are immunological intent and can be left in place in case of infection. We used PermacolTM, a collagen derived material that is gradually absorbed and replaced by the patient's own collagen.

Although the follow-up time for this patient is relatively short and the risk of recurrence increases with time, this less invasive procedure has controlled the prolapse. We found that laparoscopic pouchopexy with biological mesh, is an effective treatment for full thickness J-pouch prolapse. It was possible to dissect in correct surgical plans, while preserving intraabdominal vital organs and structures despite dense adhesions due to previous open abdominal operations.

\section{Conclusion}

In conclusion, laparoscopic pouchopexy with a biological mesh appears to be a reasonable option in the surgical management of a full-thickness prolapse of ileoanal J-pouch prolapse.

\section{Acknowledgments}

None.

\section{Conflicts of interest}

Author declares there are no conflicts of interest.

\section{Funding}

None.

\section{References}

1. Fazio VW, O'Riordain MG, Lavery IC, et al. Long-term functional outcome and quality of life after stapled restorative proctocolectomy. Ann Surg. 1999;230(4):575-584.

2. Sagar PM, Pemberton JH. Intraoperative, postoperative and reoperative problems with ileoanal pouches. Br J Surg. 2012;99(4):454-468.

3. Joyce MR, Fazio VW, Hull TT, et al. Ileal pouch prolapse: prevalence, management, and outcomes. J Gastrointest Surg. 2010;14(6):993-997.

4. Ehsan M, Isler JT, Kimmins MH, Billingham RP. Prevalence and management of prolapse of the ileoanal pouch. Dis Colon Rectum. 2004;47(6):885-888.

5. Ragupathi M, Patel CB, Ramos-Valadez DI, et al.Robotic-assisted laparoscopic "salvage" rectopexy for recurrent ileoanal J-pouch prolapse. Gastroenterol Res Pract. 2010;2010:790462.

6. Blazeby JM, Durdey P, Warren BF. Polypoid mucosal prolapse in a pelvic ileal reservoir. Gut. 1994;35(11):1668-1669.

7. Sahoo MR, Thimmegowda AK, Gowda MS. A single centre comparative study of laparoscopic mesh rectopexy versus suture rectopexy. J Minim Access Surg. 2014;10(1):18-22.

8. Smart NJ, Pathak S, Boorman P, et al.Synthetic or biological mesh use in laparoscopic ventral mesh rectopexy--a systematic review. Colorectal Dis. 2013;15(6):650-654. 